\section{Proton decay at the drip-line}

\section{Philip Woods}

WHAT determines whether a nuclear species exists? For nuclear scientists the answer to this poser is that the species should live long enough to be identified and its properties studied. This still begs the fundamental scientific question of what the ultimate boundaries to nuclear existence are. Work by Davids et al. ${ }^{1}$ at Argonne National Laboratory in the United States has pinpointed the remotest border post to date, with the discovery of proton decay from ${ }^{185} \mathrm{Bi}$, which has 24 neutrons fewer than the only stable isotope of bismuth, ${ }^{209} \mathrm{Bi}$.

Nuclear proton decay occurs when there is a severe deficit of neutrons compared with stable nuclear species. Neutrons provide the glue that prevents the nucleus blowing itself apart under the mutual electrostatic repulsion of the protons. If too many neutrons are removed from a nucleus it eventually becomes energetically unstable to the spontaneous emission of a proton, as nature protests against the extremes to which it is being pushed. This ultimate limit to nuclear stability is known as the proton drip-line.

The time taken for a proton to cross the nucleus is $\sim 10^{-22} \mathrm{~s}$, and state-of-theart techniques for detecting nuclear proton decay are limited to lifetimes in excess of $10^{-7} \mathrm{~s}$, so one might ask how it is possible to study this phenomenon. In the nuclear surface region, the combination of the attractive strong nuclear force and the repulsive electrostatic force gives rise to a potential energy barrier that the proton must penetrate for the decay to occur. If a nucleus has a large number of protons (the atomic number, $Z$ ) then this barrier can become high enough for the proton to be restrained inside it long enough for the isotope to be observed.

Proton decay therefore represents a beautifully simple example of a quantum tunnelling process in which, before emerging, the proton must traverse $\sim 100$ femtometres $\left(\sim 10^{-13} \mathrm{~m}\right)$ of the zone classically forbidden because its energy is too low. Ironically, alpha-decay - a more familiar and common nuclear decay mechanism - is inherently a much more complex process, involving the formation of ${ }^{4} \mathrm{He}$ clusters in the nuclear surface region. The simplicity of the proton decay mechanism, requiring only the emission of a single constituent nucleon, can be exploited to provide a unique window on the properties of nuclei at the extreme edge of existence. The potential energy barrier is critically influenced by the centrifugal potential experienced by the proton, which makes proton decay rates extremely sensitive to the orbital angular momentum of the proton in the host nucleus. Hence pro- ton decay half-life measurements can be used to explore nuclear shell structures in this twilight zone of nuclear existence.

The proton drip-line sounds an interesting place to visit. So how do we get there? The answer is an old one: fusion. In this case, the fusion of heavy nuclei produces highly neutron-deficient compound nuclei, which rapidly de-excite by boiling off particles and gamma-rays, leaving behind a plethora of highly unstable nuclear species. Only a small fraction of these, about $10^{-5}$ to $10^{-7}$, may be the proton-unstable isotope of interest. Ultrasensitive techniques, involving in-flight electromagnetic separation devices and sophisticated detection systems in which radioactive ions are implanted before decaying, have been developed to filter out and identify these extremely rare isotopes. Consequently, we can now explore extended regions of the proton drip-line in detail for the first time.

Particularly dramatic progress has been made in the region of odd- $Z$ elements between thulium $(Z=69)$ and gold $(Z=79)$ for which a continuous series of protondecaying isotopes has been discovered ${ }^{2,3}$. Most recently, work by Davids et al. has established the current extreme outpost for such studies with the identification of proton emission from the isotope ${ }^{185} \mathrm{Bi}$, which represents by far the heaviest point at which the drip-line has been crossed. This isotope is a particularly interesting case since it has one proton more than the magic number $Z=82$, corresponding to the closure of a major nuclear shell. In fact the observed proton decay comes from an excited intruder state configuration formed by the promotion of a proton from below the $Z=82$ shell closure. This decay transition was used to provide unique information on the quantum mixing between normal and intruder state configurations in the daughter nucleus.

Following the serendipitous discovery of nuclear proton decay ${ }^{4}$ from an excited state in 1970, and the first example of ground-state proton decay ${ }^{5}$ in 1981, there followed relative lulls in activity. Now we are entering a renaissance of proton dripline research, in which we can aim to fully

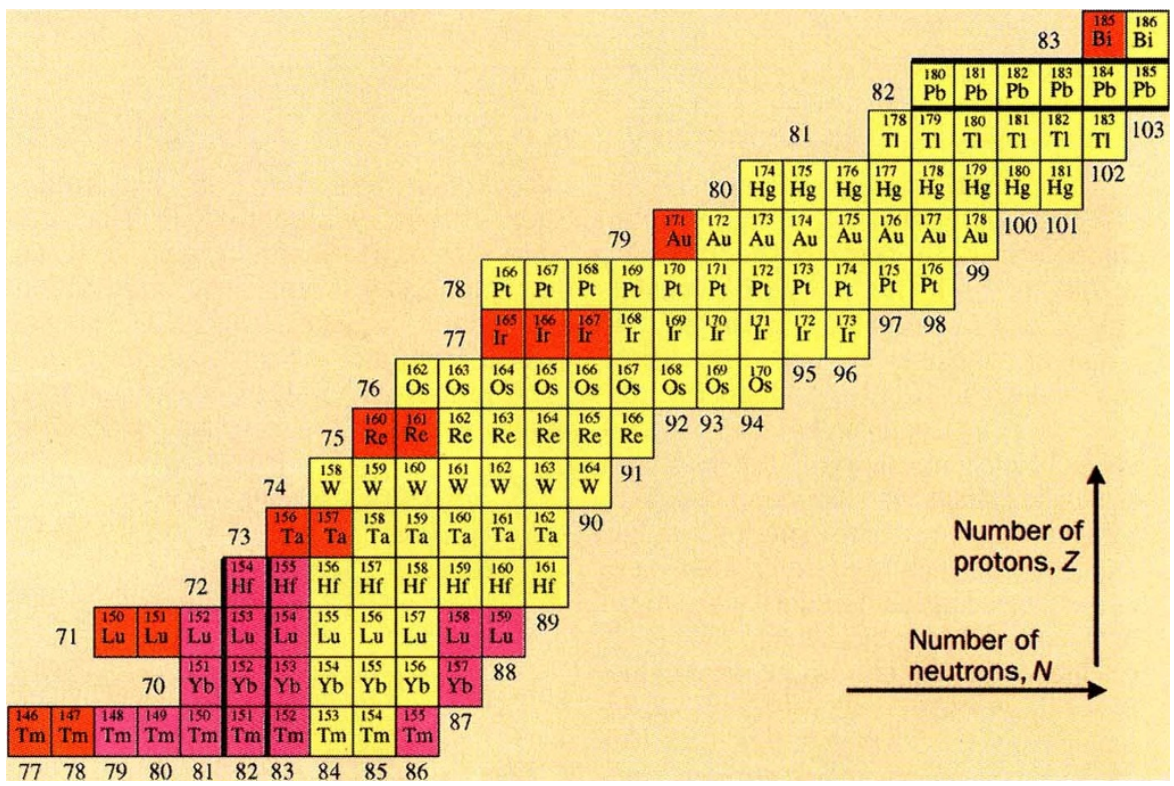

The proton drip-line, as currently established, between thulium and bismuth. The red isotopes decay by proton emission, yellow by $\alpha$ and $\beta$ only, and pink by $\beta$ only. All the proton emitters have odd $Z$, corresponding to an unpaired, energetically unbound proton. Proton emission from even- $Z$ isotopes has yet to be identified, because attractive pairing interactions push the dripline beyond current isotope production limits. In future it is hoped to observe two-proton emission from even- $Z$ nuclei, in cases where single-proton emission is energetically forbidden.

map one of the fundamental limits to nuclear stability. Furthermore, new techniques have been developed to study in detail gamma-ray de-excitation cascades from excited proton-radioactive nuclei ${ }^{6}$. With the advent of radioactive beam facilities allied to these techniques, the prospects for nuclear drip-line research look highly promising.

Philip Woods is in the Department of Physics and Astronomy, University of Edinburgh, Mayfield Road, Edinburgh EH9 3JZ, UK.

1. Davids, C. N. et al. Phys. Rev. Lett. 76, 592-595 (1996).

2. Woods, P. J. et al. Nucl. Phys. A553, 485-488 (1993).

3. Davids, C. N. et al. Proc. Exotic Nuclei and Atomic Masses Conf., Arles, 1995 (in the press).

4. Jackson, K. P. et al. Phys. Lett. B33, 281-284 (1970).

5. Hofmann, S. et al. Proc. 4th International Conf. Nuclei far from Stability CERN 81-09, 190-201 (1981). 6. Paul, E. S. et al. Phys. Rev. C51, 78-87 (1995) 